\title{
CSF1R Inhibitor DCC-3014
}

National Cancer Institute

\section{Source}

National Cancer Institute. CSF1R Inhibitor DCC-3014. NCI Thesaurus. Code C136424.

An orally bioavailable inhibitor of the tyrosine kinase receptor colony stimulating factor 1 receptor (CSF1R; CSF-1R; C-FMS; CD115; M-CSFR), with potential antineoplastic, macrophage checkpoint-inhibitory and immunomodulating activities. Upon administration, CSF1R inhibitor DCC-3014 targets and binds to CSF1R expressed on monocytes, macrophages, and osteoclasts and inhibits the binding of the CSF1R ligands colony-stimulating factor-1 (CSF-1) and interleukin-34 (IL-34), to CSF1R. This prevents CSF1R activation and CSF1R-mediated signaling in these cells. This blocks the production of inflammatory mediators by macrophages and monocytes and reduces inflammation. By blocking the recruitment to the tumor microenvironment and activity of CSF1Rdependent tumor-associated macrophages (TAMs), DCC-3014 inhibits the immunomodulating activity by macrophages and enhances T-cell infiltration and antitumor T-cell immune responses, which inhibits the proliferation of tumor cells. TAMs play key roles in the tumor microenvironment and allow for immune suppression; TAMs promote inflammation, tumor cell proliferation, angiogenesis, invasiveness and survival. 